This material is published in the open archive of Mid Sweden University DIVA http://miun.divaportal.org to ensure timely dissemination of scholarly and technical work.

Copyright and all rights therein are retained by authors or by other copyright holders. All persons copying this information are expected to adhere to the terms and constraints invoked by each author's copyright. In most cases, these works may not be reposted without the explicit permission of the copyright holder.

Damghanian, M.; Olsson, R.; Sjöström, M.; Erdmann, A.; Perwass, C., "Spatial resolution in a multifocus plenoptic camera," IEEE International Conference on Image Processing (ICIP 2014), vol., no., pp.1932-1936, 27-30 Oct. 2014.

(C) 2014 IEEE. Personal use of this material is permitted. However, permission to reprint/republish this material for advertising or promotional purposes or for creating new collective works for resale or redistribution to servers or lists, or to reuse any copyrighted component of this work in other works must be obtained from the IEEE. 


\section{SPATIAL RESOLUTION IN A MULTI-FOCUS PLENOPTIC CAMERA}

\author{
M. Damghanian, R. Olsson, M. Sjöström
}
Department of Information and Communication Systems, Mid Sweden University, Sundsvall, Sweden

\author{
A. Erdmann, C. Perwass
}

Raytrix GmbH
Kiel, Germany

\begin{abstract}
Evaluation of the state of the art plenoptic cameras is necessary for design and application purposes. In this work, spatial resolution is investigated in a multi-focus plenoptic camera using two approaches: empirical and model-based. The Raytrix R29 plenoptic camera is studied which utilizes three types of micro lenses with different focal lengths in a hexagonal array structure to increase the depth of field. The modelbased approach utilizes the previously proposed sampling pattern cube (SPC) model for representation and evaluation of the plenoptic capturing systems. For the experimental resolution measurements, spatial resolution values are extracted from images reconstructed by the provided Raytrix reconstruction method. Both the measurement and the SPC model based approaches demonstrate a gradual variation of the resolution values in a wide depth range for the multi focus R29 camera. Moreover, the good agreement between the results from the model-based approach and those from the empirical approach confirms suitability of the SPC model in evaluating high-level camera parameters such as the spatial resolution in a complex capturing system as R29 multi-focus plenoptic camera.
\end{abstract}

Index Terms- Plenoptics, spatial resolution, camera evaluation

\section{INTRODUCTION}

Plenoptic cameras provide a novel means for capturing 3D in less controlled environments and in a single snapshot $[1,2]$. They make 3D capture of the live scene possible by compromising the spatial resolution to gain the depth information [3]. The resolution of the currently available image sensors can surpass the required resolution in many applications, and then plenoptic cameras provide the chance to use this extra resolution budget on gaining the depth information about the scene, opening the gateway to a broad range of applications such as single snapshot 3D, refocusing and extended depth of field, naming a few.

This work has been supported by grant 2009/0264 of the KK Foundation, Sweden, by grant 00156702 of the EU European Regional Development Fund, Mellersta Norrland, Sweden, and by grant 00155148 of Länsstyrelsen i Västernorrland, Sweden, and by ECOST Action IC1105.

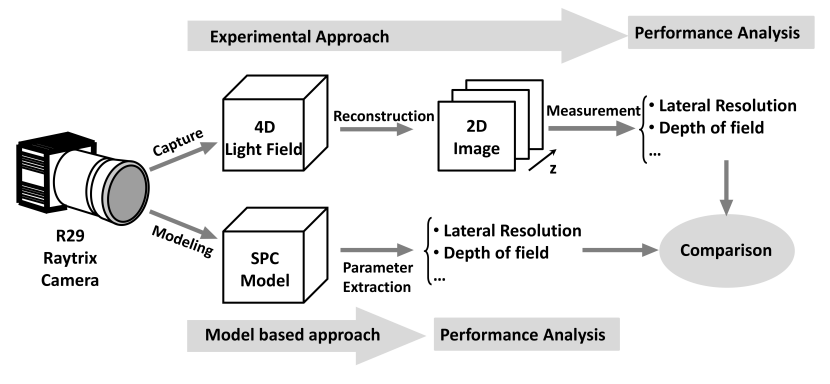

Fig. 1. The experimental and the model-based methods for the performance analysis of the R29 plenoptic camera

Evaluation of the state of the art plenoptic cameras is necessary for both the camera manufacturers and the users to look into the camera properties accordingly. Fast and accurate camera evaluation techniques will facilitate the usage of the plenoptic cameras in proper application areas as well as providing a tool for the camera designers and manufacturers to optimize the camera for specific applications.

Evaluation of the plenoptic cameras as complex capturing systems is more extensive than their conventional counterparts. Plenoptic cameras introduce strong trade-offs between the high-level camera parameters such as different resolution terms. The strong interconnection between the parameters such as spatial and angular resolution, depth of field and refocusing ability makes the traditional measurement methods exhaustive and sometimes even out of reach, especially for the camera users. Fast and reliable model-based evaluation approaches will provide a straight forward means for evaluation of the camera parameters in the multi-dimensional camera parameter space.

Models have been introduced that describe the lightfield and how it is sampled by different image capturing systems $[4,5]$. The lightfield model has proven useful for applications spanning computer graphics, digital photography, and 3D reconstruction [6], but to our best knowledge, has not been utilized for extraction of the high-level camera properties. Attempts are made for model-based extraction of the high-level camera properties such as spatial resolution, using the principal ray model, the previously proposed SPC model and the complete wave-optics-based simulations [7, 8]. In each case, 


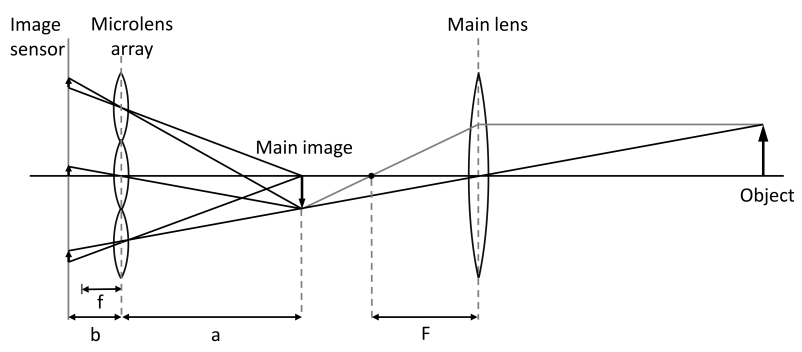

Fig. 2. Basic structure of a focused plenoptic camera

the level of computational complexity and the result accuracy corresponds to the utilized model. The SPC model, which describes the captured light elements in a camera system in the form of the light cones (LCs), has been proven useful in describing the light sampling properties of a number of complex capturing systems $[7,8]$. The model has been previously utilized for spatial resolution analysis of a synthetic aperture integral imaging setup [8] and a plenoptic capturing setup [9]. The SPC model is in the scope of the geometrical optics models, yet contrary to the principal ray model, it carries the focus properties of the optical system in addition to the ray information. This property makes the SPC model capable of extracting high-level properties of the capturing system, like the spatial resolution, with better accuracy compared to the ray-based models, and lower level of complexity compared to the wave-optics model.

The novelty of the current work lies in using the SPC model for performance analysis of a multi-focus plenoptic camera. In this work we investigate the spatial resolution in Raytrix R29 camera using two approaches: empirical and model-based (see Figure 1). Raytrix R29 camera is a highend plenoptic camera currently available in the market, with a $29 \mathrm{Mpx}$ image sensor. Here the spatial resolution profile of R29 is quantified as a function of depth. The model-based approach is using the previously developed SPC model $[7,10]$.

In the next section, the structure of the plenoptic camera in general and the R29 camera in particular are introduced, followed by the description of the method for camera performance analysis, using the model-based and the experimental approaches. Results are illustrated and discussed in the subsequent section and finally the work is concluded.

\section{PLENOPTIC CAMERA STRUCTURE}

To make a plenoptic camera, a micro lens array (MLA) structure is introduced to a conventional camera system to empower capturing the directional information. The general plenoptic camera setup is shown in Figure 2. The main lens generates an image which is then projected by the micro lenses onto the image plane.

Based on whether the image sensor collects a focused or blurry projection of the main lens image, the resulting cam-

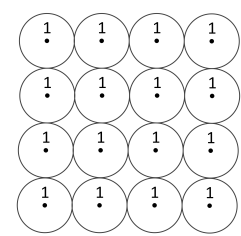

(a)

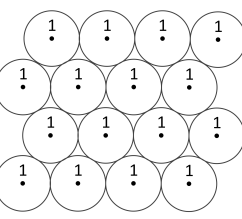

(b)

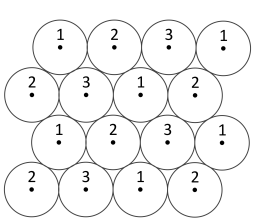

(c)
Fig. 3. Various MLA arrangements (a) orthogonal with single lens type (b) hexagonal, giving a better fill factor (c) hexagonal with three lens types each having a different focal length

era is named a conventional or a focused plenoptic camera. Note that the image may also be virtual in the sense that it is formed behind the image plane. The virtual image setup helps to reduce the size of the plenoptic camera and hence is usually the preferred setup, as in Raytrix R29 camera as well. In a focused plenoptic camera, each micro lens is re-imaging the object from a slightly different perspective, which is in fact spending the spatial resolution budget in respect to a conventional camera and gaining the angular information, based on the disparity between neighbouring micro images.

The MLA in a plenoptic camera can have an orthogonal, hexagonal (with an improved fill factor) or other possible arrangements (see Figure 3). Geometry, placement and the quality of the MLA strongly affects the properties of the plenoptic camera.

The Raytrix R29 camera utilizes a 2D micro lens array consisting of three interlaced hexagonal MLAs each having a different focal length. The focal lengths of the micro lenses are chosen such that the depth of field (DoF) of different micro lens types just touch and hence extend the overall DoF of the R29 multi-focus plenoptic camera [11]. Because of the complex structure of the plenoptic cameras in general and the multi-focus plenoptic cameras in particular, simplifying assumptions and approximations are employed for extracting high-level properties of these cameras using the analytical approach [11].

\section{METHOD}

The profile of the spatial resolution throughout various depth planes is an indicator of the performance of the plenoptic camera, which empowers us to make a comparison between various camera structures with different capture parameters. Here we investigate the spatial resolution profile of the camera as a function of the distance to the image sensor. Figure 1 illustrates steps in R29 camera evaluation in both empirical and the model-based approaches.

For the scope of this work, the spatial resolution $R$ for a plenoptic capturing system is defined as the inverse of the minimum distance between two resolvable points $x_{1 z}$ and $x_{2 z}$ located at a specific depth plane $z$ perpendicular to the optical 
axis. $R$ is then normalized with respect to the sensor pixel pitch $p$ giving the normalized spatial resolution $R_{n}$ as:

$$
R_{n}=\frac{p}{\min \left[x_{1 z}-x_{2 z}\right]} .
$$

The depth plane of interest can be located in the system's common field of view (CFoV) or be visible for only a limited number of lens elements in the system.

The depth of field $(D o F)$ is defined as the depth range in which the rendered image is sharp with respect to a resolution threshold $R_{t h}$ :

$$
\operatorname{DoF}\left(R_{t h}\right)=D_{f a r}\left(R_{t h}\right)-D_{\text {near }}\left(R_{t h}\right) .
$$

Thus the $D o F$ value depends on the choice of the resolution threshold that separates the depth ranges which give sharp (in focus) and blurry (out of focus) images. It is important to have the same resolution threshold when comparing the $D o F$ between different capturing systems.

\subsection{SPC Model-Based Approach}

The SPC model is defined as a set of light samples in the form of light cones (LCs) each having a tip position and an angular span. To build the SPC model of a capturing system, first the light intensity captured in each image sensor pixel is described in the form of a light cone. In the next step all the light cones are projected into the scene, passing through the optical elements in the capturing system and ending up in a new set of light cones with new spans and tip positions, which is then called the SPC model of the capturing system. The SPC model carries the ray information and the focus information of the capturing system and describes where within the scene the captured data set originates from [10]. The SPC model then uses this information to extract camera parameters such as spatial resolution throughout depth [7].

The SPC model-based spatial resolution extractor defined in [7] is valid for the area within the CFoV of the capturing system. To guarantee the resolvability of two points on a single depth plane in the $\mathrm{CFoV}$, the minimum distance between the two points should be greater or equal to $p_{a}+\alpha$, where $p_{a}$ is the projected pixel size and $\alpha$ is the biggest line piece created by the LC's bases in the CFoV (see Figure 4). So Equation (1) turns into:

$$
R_{n}=\frac{p}{p_{a}+\alpha},
$$

where the values for $p_{a}$ and $\alpha$ in the depth plane of interest are obtained from the SPC model of the capturing system. We use an incremental methodology to use the same extractor for the depth ranges which are visible only by a limited number of the micro lenses. For this purpose, the depth range of interest is divided into smaller depth ranges which are in the $\mathrm{CFoV}$ of not all but a number of micro lenses. For each depth range, the spatial resolution is then obtained by using

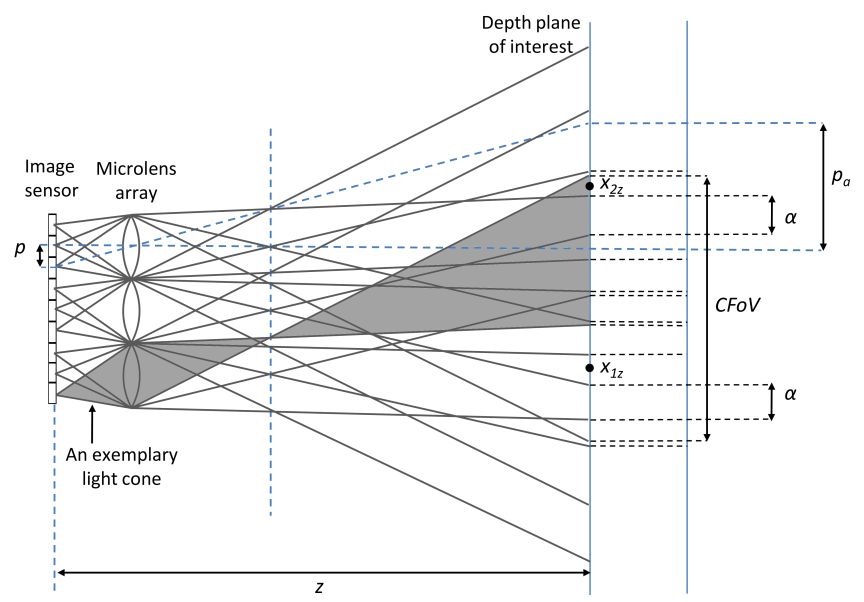

Fig. 4. Illustrating parameters for extracting the spatial resolution in the SPC model of the capturing system

the same extractor in the proper $\mathrm{CFoV}$ related to that number of micro lenses. The results will be stitched together to cover the whole depth range of interest. A more detailed description of this incremental methodology can be found in [9].

The SPC model of the R29 camera is generated with the method described in [10] using the exact geometry of the optical components including the image sensor and the interlaced micro lens array properties and spacing. Using Equation (3) and the described incremental method, the spatial resolution is extracted from the SPC model of the camera for the depth range of interest. Considering the periodic nature of the R29 camera structure, we look into the model of the system for a single line of pixels behind a single line of micro lenses to extract the spatial resolution profile.

\subsection{Experimental Approach}

The experimental setup incorporated an R29 monochrome camera with $100 \mathrm{~mm}$ Zeiss lens capturing a scene with a costumed $80 \times 80$ checkered test chart rotated with 5 degrees, and a motorized camera slider. The main lens was focused at $80 \mathrm{~cm}$ and the distance between the camera and the test chart was varied from $60 \mathrm{~cm}$ to $90 \mathrm{~cm}$. A set of 3000 so called lightfield photos was acquired using the R29 camera at uniformly spaced depths. The camera was operated with fixed focus and zoom in the whole depth range. Refocused image stacks were produced from each lightfield photo using the Rxlive software. The image in each stack exhibiting the highest sharpness was selected as having a focus plane coinciding with the test chart and used in subsequent resolution analysis by measuring the systems modulation transfer function (MTF) as set out in [12]. The measure of MTF50 was used which resembles the perceived sharpness of an image and corresponds to the spatial frequency of the MTF equal to a contrast of $50 \%$ relative to that of the MFT at spatial 


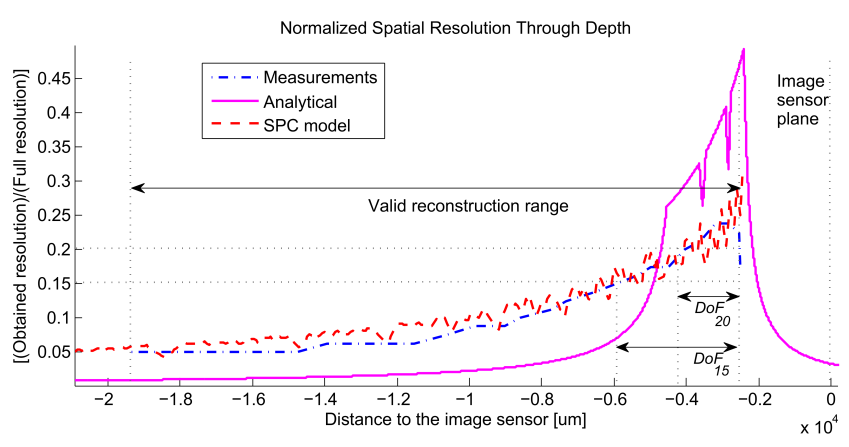

Fig. 5. Normalized spatial resolution for R29 camera from empirical, analytical and SPC model-based approaches

frequency 0 . Obtained MTF50 values were then normalized with respect to $M T F 50=0.5 \mathrm{cycle} / \mathrm{pixel}$ corresponding to the full sensor resolution.

\section{RESULT AND DISCUSSION}

Figure 5 illustrates the spatial resolution profile from the model-based and the empirical approaches, along with the results from the analytical approach [11] for comparison purposes.

Horizontal axis in Figure 5 shows the distance of the main lens image to the image sensor, and the negative values on the axis come from the virtual image setup of the R29 camera (see section 2). The capture range defined as the distance of the object to the camera's main lens $(60 \mathrm{~cm}$ to $90 \mathrm{~cm}$ in the capturing setup) is mapped through the main lens to the range $2.5 \mathrm{~mm} \leq z \leq 19.5 \mathrm{~mm}$ on this axis. Getting close to the margins of this depth range, the best focus images used for MTF measurements exhibit reconstruction artifacts, which means the chosen depth values covers the whole working range of Rxlive reconstruction capabilities for the current capture settings.

The vertical axis in Figure 5 represents the spatial resolution normalized with respect to the full sensor resolution. Figure 5 shows that the spatial resolution results from the SPC model based and the empirical approaches are in a very good agreement, yet both deviate from the results from the analytical approach, which predicts a higher maximum and then a sharp fall for the spatial resolution. The maximum obtainable spatial resolution from the SPC model based and the empirical approaches is shown to be approximately a quarter of the image sensor resolution in one dimension. Both the model-based and the analytical approaches assume an ideal main lens in the results shown in Figure 5. It should be noted that the same camera without the MLA may not reach the full sensor resolution or the normalized value of 1 because of the main lens imperfections and diffraction limits. In that case, spatial resolution values normalized with respect to the maximum amount of the achievable resolution in the conventional camera will represent the amount of resolution loss as the result of introducing the MLA to the camera system.

One reason for the difference between the resolution results from the analytical approach with those from the measurements and the model-based approach is the fact that achievable resolution of different lens types are not combined in the analytical approach. In the analytical approach, the resolution value for each depth is selected by looking into the best resolution that each type of the micro lenses in the MLA can provide [11], rather than a combination of them. In contrast, in the SPC model based method as well as the rendered in focus images used for resolution measurements, all lens types contribute to the resolution values. The contribution of different lens types smooths the resolution graph, lowering the maximum peak but giving a less steep fall off of the resolution values compared to what is predicted in the analytical approach. As a result, both the model-based and the empirical approaches show a smooth change in the spatial resolution values through depth, giving a narrower $D o F$ for higher resolution thresholds and a wider $D o F$ for lower resolution thresholds. As an example, Figure 5 illustrates that the $D_{o F}$ values for resolution thresholds equal to 0.2 and 0.15 (on the normalized resolution axis) are correspondingly narrower and wider than the analytically predicted amounts.

\section{CONCLUSION}

The spatial resolution profile for different depths was investigated in the R29 multi-focus plenoptic camera, using empirical and the SPC model based approaches. Maximum obtainable spatial resolution is approximately a quarter of the image sensor resolution in one dimension. Results from both measurements and the SPC model based approach are in a very good agreement. Obtained results show a lower maximum but a smoother change in the spatial resolution profile compared to the analytically predicted values. Both the empirical and the SPC model based approaches show stable spatial resolution values in a wide depth range for the multi-focus R29 camera. The good agreement between the results from the model-based approach and those from the empirical approach indicates suitability of the SPC model in evaluating high-level camera parameters such as the spatial resolution in a complex capturing system as R29 multi-focus plenoptic camera. This model-based approach has proved to be straight forward and applicable to plenoptic cameras with various configurations including the multi-focus MLA structure.

\section{REFERENCES}

[1] Gabriel Lippmann, "Epreuves reversibles. photographies integrals," Comptes-Rendus Academie des Sciences, vol. 146, pp. 446-451, 1908. 
[2] Ren Ng, Digital light field photography, Ph.D. thesis, stanford university, 2006.

[3] Todor Georgiev, Colin Zheng, Brian Curless, David Salesin, Shree Nayar, and Chintan Intwala, "Spatioangular resolution tradeoffs in integral photography," in Eurographics Symposium on Rendering, 2006, pp. 263272.

[4] Steven J. Gortler, Radek Grzeszczuk, Richard Szeliski, and Michael F. Cohen, "The lumigraph," in Proceedings of the 23rd annual conference on Computer graphics and interactive techniques, New York, NY, USA, 1996, SIGGRAPH '96, pp. 43-54, ACM.

[5] Marc Levoy and Pat Hanrahan, "Light field rendering," in Proceedings of the 23rd annual conference on Computer graphics and interactive techniques, New York, NY, USA, 1996, SIGGRAPH '96, pp. 31-42, ACM.

[6] Marc Levoy, "Light fields and computational imaging," Computer, vol. 39, no. 8, pp. 46-55, Aug. 2006.

[7] Mitra Damghanian, Roger Olsson, and Mårten Sjöström, "Extraction of the lateral resolution in a plenoptic camera using the SPC model," in $3 D$ Imaging (IC3D), 2012 International Conference on, 2012, pp. $1-5$.

[8] Mitra Damghanian, Roger Olsson, Mårten Sjöström, Hector Navarro Fructuoso, and Manuel MartinezCorral, "Investigating the lateral resolution in a plenoptic capturing system using the SPC model," in $I S \& T / S P I E$ Electronic Imaging. International Society for Optics and Photonics, 2013, pp. 86600T-86600T.

[9] Mitra Damghanian, Roger Olsson, and Mårten Sjöström, "Performance analysis in lytro camera: Empirical and model based approaches to assess refocusing quality," in Acoustics, Speech and Signal Processing (ICASSP), 2014 IEEE International Conference on, May 2014.

[10] Mitra Damghanian, The Sampling Pattern Cube : A Framework for Representation and Evaluation of Plenoptic Capturing Systems, Number 99 in Mid Sweden University licentiate thesis. Mid Sweden University, 2013.

[11] Christian Perwass and Lennart Wietzke, "Single lens 3D-camera with extended depth-of-field," in IS\&T/SPIE Electronic Imaging. International Society for Optics and Photonics, 2012, pp. 829108-829108.

[12] "Photography - Electronic still-picture cameras - Resolution measurements," 2000. 